Ann. Biol. anim. Bioch. Biophys., 1971, 11 (4), 681-694.

\title{
ACTION POST MORTEM DU PH ET DE LA TEMPÉRATURE SUR LE GAPTAGE DE GALCIUM ET L'ACTIVITÉ ATPASIQUE DU RÉTICULUM SARCOPLASMIQUE FRAGMENTÉ DU MUSCLE DE BOVIN
}

\author{
A. LACOURT \\ avec la collaboration technique de Marie-Christine BAYLE \\ Station de Recherches sur la Viande, \\ Centre de Recherches de Clermont-Ferrand, I. N. R. A., \\ 63 - Saint-Genès-Champanelle
}

\section{RÉSUMÉ}

Le captage du calcium par le réticulum sarcoplasmique fragmenté, prélevé sur le muscle Rectus abdominis de bovin, diminue régulièrement avec l'acidification post mortem, alors que l'activité ATPasique reste constante. La fixation du calcium est totalement inhibée par un $\mathrm{pH}$ inférieur à 5,8 ou supérieur à 7,7 et par une température inférieure à $+5^{\circ} \mathrm{C}$ quel que soit le $\mathrm{pH}$. L'incubation de réticulum sarcoplasmique fragmenté dans du tampon Tris maléate o, I Mà des températures comprises entre $10^{\circ} \mathrm{C}$ et $40^{\circ} \mathrm{C}$ et à des $\mathrm{pH}$ compris entre 5,5 et 7,3 inhibe le captage du calcium et multiplie l'hydrolyse de l'ATP 3 à 8 fois. L'aptitude à fixer le calcium est mieux conservée à $30-35^{\circ} \mathrm{C}$ et à $\mathrm{pH} 6,5-7, \mathrm{O}$. L'action d'une activité protéolytique au cours de l'incubation est discutee.

\section{INTRODUCTION}

Depuis les travaux de SzENT Györgyr (I947), il est admis que la contraction musculaire est liée à l'activité ATPasique de la myosine. Cette activité ne peut se manifester que lorsque le taux de calcium dans le sarcoplasme atteint la valeur de Io $^{-6} \mathrm{M}$. En présence d'ATP (') le calcium est accumulé dans le réticulum sarcoplas-

(') Abréviations employées :

RSF : réticulum sarcoplasmique fragmenté

ATP : adénosine triphosphate,

ATPase : adénosine triphosphate phosphohydrolase. 
mique au moyen d'un transport actif membranaire. Il n'est relargué dans la cellule que lorsqu'une excitation nerveuse provoque une dépolarisation générale des membranes. La préparation et l'étude du réticulum sarcoplasmique fragmenté, appelé grana ou RSF, ont fait l'objet de nombreux travaux dans le cas du muscle vivant. EBASH (I96I), Hasselbach et MaKinOSE (I96I) ont mis en évidence que le RSF de muscles squelettiques capte le calcium d'une façon remarquable en présence de $\mathrm{Mg}^{++}$ et d'ATP. MARTONOSI et FERETOS (I964) ont confirmé que la présence d'oxalate diminue 1'activité ATPasique et augmente le captage de calcium. En effet, la précipitation du calcium sous forme d'oxalate à l'intérieur des granas évite sa fuite vers le milieu extérieur. SAMAHA et GERGELY (I965) ont montré que le RSF issu des fibres blanches de type rapide a une activité ATPasique et une capacité de captage du $\mathrm{Ca}^{++}$ supérieures à celles des fibres rouges de type lent. Cette étude a été reprise par HaRIGAYA, OGAWA et SugiTa (I968) qui ont montré que si le réticulum sarcoplasmique est moins abondant dans les fibres rouges, il y joue très certainement le même rôle que dans les fibres blanches.

Post mortem, la première phase de l'évolution du muscle en viande, l'installation de la rigor mortis est caractérisée par 1'hydrolyse des composés riches en énergie, phosphocréatine et ATP. La disparition de ces composés se traduit au niveau moléculaire par la combinaison de l'actine et de la myosine et la fixation de l'appareil myofibrillaire dans un état plus ou moins contracté. D'une façon générale il semble qu'une installation très rapide de l'état de rigor soit préjudiciable à l'élaboration des qualités organoleptiques des viandes. GREASER et al. (Ig67) puis CHARPENTIER (I97I) ont montré chez le Porc, qu'une installation rapide de la rigor mortis était liée à une perturbation du fonctionnement du RSF dès les premiers instants qui suivent l'abattage. Aucun travail important n'a encore été fait dans le cas particulier des bovins, bien que ces phénomènes puissent avoir une incidence pratique très importante dans le muscle de taurillon ou lors de la contracture au froid déclenchée par une réfrigération trop rapide. La présente étude a donc été entreprise dans le but d'éclaircir le fonctionnement du RSF du muscle de bœuf.

\section{MATÉRIEL, ET MÉTHODES}

$$
\text { I. - Matériel }
$$

L'étude a porté sur des animaux de différentes races, comprenant d'une part des vaches de 4 à 5 ans, d'autre part des boufs de 2 à 4 ans. Les animaux avaient bénéficié d'un repos suffisant avant l'abattage, qui a été effectué aux abattoirs du C. R. Z.V. de Theix. Les prélèvements ont été faits 30 minutes après la mort sur le muscle Rectus abdominis (bavette de flanchet).

\section{Extraction des granas.}

\section{2. - Methodes}

Toutes les opérations d'extraction ont été effectuées à une température inférieure ou égale $\mathrm{a}^{*}+5^{\circ} \mathrm{C}$ selon la méthode de SAmafa et GERGELy (1965) qui a été légèrement modifiée pour ce travail. Cent grammes de muscle sont homogénéisés au broyeur Biorex pendant I minute dans $400 \mathrm{ml}$ de saccharose $0,25 \mathrm{M}$, histidine $5 \mathrm{mM}, \mathrm{pH} 7,3$. Un essai préliminaire nous a en effet montré qu'une durée de broyage inférieure à I minute est insuffisante, et que si elle excède 2 minutes, la suspension de RSF finalement recueillie contient une quantité importante de fragments de myofi- 
brilles. Deux centrifugations successives de 20 minutes à I $500 \mathrm{~g}$ puis à $\mathbf{I} 4000 \mathrm{~g}$ ont permis d'éliminer le conjonctif et les myofibrilles, puis les mitochondries. Les matières grasses sont séparées par filtration sur 6 épaisseurs de gaze. La fraction lourde du RSF est lavée et recueillie par centrifugation à 33 ooo $g$ pendant I heure. Le culot est alors homogénéisé doucement dans $4 \mathrm{ml}$ de saccharose-histidine à l'aide d'un tube de Potter. Puis il est réparti en fractions de $\mathrm{r}$ ml et congelé par immersion dans l'azote liquide. Dans ces conditions Charpentier (I97I) a mis en évidence sur granas de porcs qu'il était possible de les conserver à - $30^{\circ} \mathrm{C}$. et de les utiliser jusqu'à 20 jours après leur préparation sans que leur activité soit diminuée.

La teneur en protéines a été déterminée par la réaction du Biuret en utilisant comme gamme étalon une solution de sérumalbumine bovine fraction 4 .

Caractérisation de la glycogénolyse post mortem.

Le $\mathrm{pH}$ a été mesuré à l'aide d'un pHmètre Radiometer par contact direct de l'électrode humide avec le muscle incisé transversalement.

L'acide lactique et l'ATP ont été dosés à l'aide de produits Biotrol selon les techniques classiques de BerGmeyer (I963) dans des extraits de muscle déprotéinisés par l'acide perchlorique $0,6 \mathrm{M}$.

\section{Mesure du captage de calcium et de l'activité ATPasique du RSF.}

Le milieu final d'incubation est constitué par une solution de $\mathrm{KCl}$ o, $\mathrm{I} \mathbf{M}$ tamponnée aux $\mathrm{pH}$ désirés et contenant en outre $\mathrm{MgCl}_{2} 5 \mathrm{mM},{ }^{45} \mathrm{CaCl}_{2} 0,02 \mathrm{mM}$ (activité volumique $0, \mathrm{I}_{5}$ à 0,25 $\mu \mathrm{Ci} / \mathrm{ml}$ ), oxalate de $\mathrm{K} 5 \mathrm{mM}$, ATP disodique $5 \mathrm{mM}$. Après avoir amené le milieu d'incubation à la température de l'expérience, la réaction est déclenchée par l'addition de granas à la concentration finale de 0,04 à $0,05 \mathrm{mg}$ de protéines par $\mathrm{ml}$. Des fractions aliquotes de $2 \mathrm{ml}$ prélevées en fonction du temps ont servi à déterminer d'une part le captage de calcium et d'autre part l'activité ATPasique.

a) Captage du calcium.

Le RSF est séparé de la phase liquide par filtration à travers un filtre Millipore type HA ayant des pores de diamètre moyen de 0,45 micron, et la radioactivité du filtrat et des granas retenus sur le filtre a été mesurée dans un compteur à scintillation liquide Tricarb 3375 Packard. Le standard extérieur ou AES est resté constant au cours de cette étude. Il était égal à o,87 pour les filtres immergés dans du toluène et à 0,61 pour les filtrats émulsionnés dans un mélange toluènetriton 5 volumes pour $\mathrm{I}$ volume.

\section{b) L'activité ATPasique.}

Elle a été déterminée en dosant par la méthode de Fiske et Subbarow le phosphore inorganique libéré dans le milieu d'incubation. Une déprotéinisation préalable au dosage a été obtenue par addition d'acide trichloracétique à la concentration finale de $3 \mathrm{p}$. roo.

Tous les produits étaient de qualité réellement pure pour analyses. L'ATP provenait de la firme $\mathrm{N}$. B. C. et le ${ }^{45} \mathrm{CaCl}$ du Commissariat à l'Énergie atomique, son activité spécifique était de $210,8 \mathrm{mCi} / \mathrm{g}$.

\section{RÉSULTATS}

Les extractions ont conduit à l'obtention de suspensions de granas dont la concentration en protéines varie de o,I à $0,35 \mathrm{mg}$ par gramme de muscle.

\section{I. - Captage de calcium et activité ATPasique des granas de muscle de bovin}

Le RSF de muscle de bovin s'avère capable en présence de $\mathrm{Mg}^{++}$, ATP et oxalate de fixer rapidement le calcium. La figure I représente le captage pour des concentrations de I7,5 $\mu \mathrm{M}$ et $25 \mu \mathrm{M}$ de calcium dans le milieu d'incubation. La courbe a une pente très rapide dans ces deux cas pendant les deux premières minutes puis plafonne 
à des valeurs qui dépendent de la quantité de calcium dans le milieu extérieur. Pour une même concentration de RSF, le maximum atteint en présence de calcium I7,5 $\mu \mathrm{M}$ est de $0,39 \mu$ mole de calcium fixé/mg de protéine et en présence de $\mathrm{Ca}^{++} 25 \mu \mathrm{M}$ il est de $0,53 \mu$ mole de $\mathrm{Ca}^{++} / \mathrm{mg}$ protéine. Ainsi 95 à $98 \mathrm{p}$. roo du calcium libre est

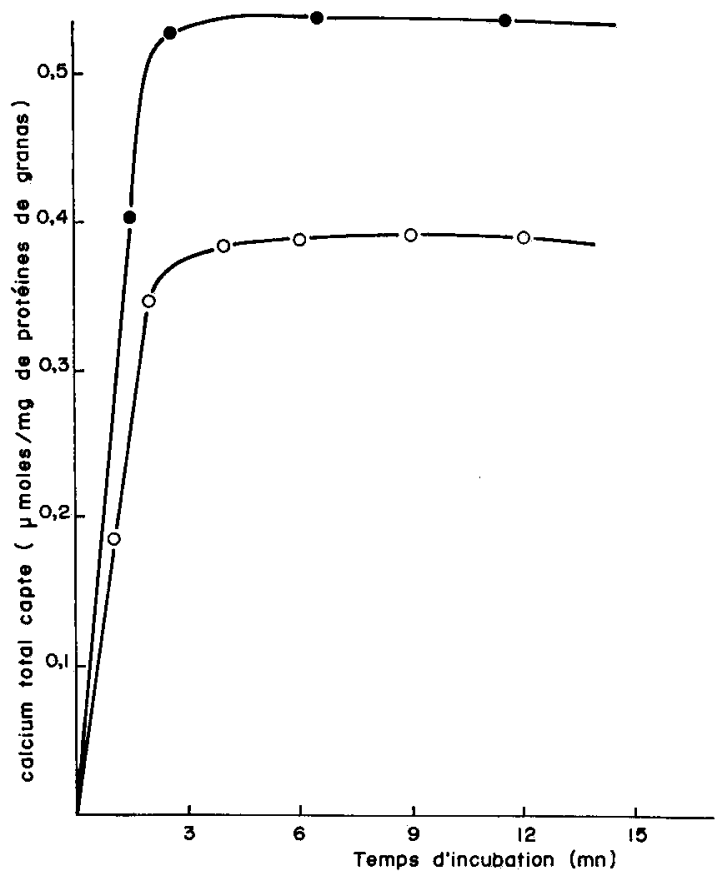

FIG. I. - Captage du calcium par le RSF de bovin

Les incubations sont faites à $\mathrm{pH} 7,3$ et à $30^{\circ} \mathrm{C}$ 0 - $1,75 \cdot 10^{-5} \mathrm{M}$ en calcium total $\bullet 2,50 \cdot 10^{-5} \mathrm{M}$ en calcium total

fixé par les granas. Ces résultats confirment ceux auxquels sont parvenus MaRToNOSI et FERETOS (I964) et HARIGAYA et al. (I968) en prélevant des muscles de lapin ou de rat aussitôt après la mort. Avec des concentrations différentes de calcium dans le milieu d'incubation, ces auteurs obtiennent un pourcentage de $\mathrm{Ca}^{++}$capté analogue à celui que nous obtenons. Le maximum de calcium capté par le RSF est fonction de la concentration du calcium dans le milieu d'incubation. Il semble donc préférable de se baser sur la vitesse initiale de captage du calcium pour apprécier l'état de fonctionnement des granas dans des conditions différentes. Le RSF du muscle Rectus abdominis de bovin étudié dans ce travail permet selon les animaux et les prélèvements de capter 0,18 à $0,30 \mu$ mole de calcium $/ \mathrm{mg}$ protéine/minute.

Les activités ATPasiques obtenues à partir des mêmes incubations en présence de calcium I7,5 et $25 \mu \mathrm{M}$ sont présentées dans la figure 2 . Au cours des deux premières minutes pendant lesquelles se produit la fixation du calcium, la vitesse de libération du phosphore inorganique ( $\mathrm{I} u \mathrm{~mole} / \mathrm{mg}$ protéine $/ \mathrm{mn}$ ) est 5 fois plus grande que celle qui existe au-delà de 3 minutes. 


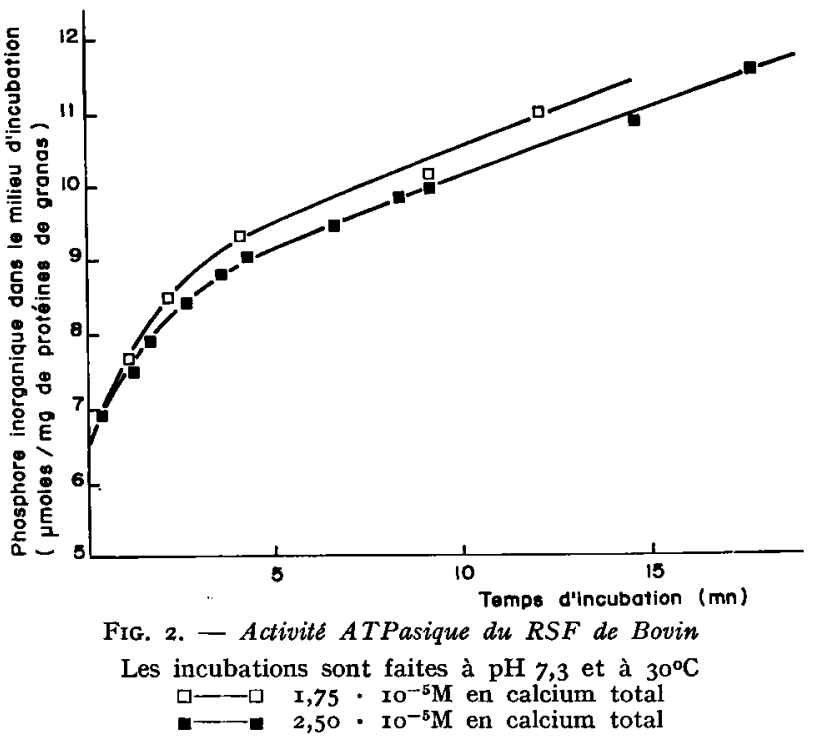

\section{2. - Influence de la glycogénolyse post mortem} sur les propriétés du RSF de bovin

Cette étude préliminaire est réalisée sur le Rectus abdominis, le Psoas major et le Diaphragma de quatre bœufs différents. L'installation de la rigor mortis est suivie en mesurant l'évolution du $\mathrm{pH}$, de l'acide lactique et de l'A'TP dans le muscle. Simultanément les granas sont extraits à I heure, 4heures, 8 heures et 24 heures post mortem. Leur capacité à capter le calcium et à hydrolyser l'ATP est alors testée à la température d'incubation de $30^{\circ} \mathrm{C}$. Au cours de l'installation de la rigor mortis, pendant que la température s'abaisse dans le muscle, on constate dans le tableau I la diminution de la concentration d'ATP et l'augmentation corrélative de la concentration en acide lactique. Celle-ci est elle-même normalement liée à l'évolution du pH vers les valeurs acides en fonction du temps. La vitesse initiale de captage de calcium diminue avec le temps post mortem. L'activité ATPasique du RSF reste le plus souvent constante et même augmente dans le cas du bœuf $I$.

La capacité de captage de calcium des granas est fonction du temps d'action du couple température-pH acide dans le muscle post mortem. En effet, si le pH dans le muscle atteint la valeur 6,0 alors que la température est supérieure à ${ }^{\circ}{ }^{\circ} \mathrm{C}, 1 \mathrm{e}$ RSF extrait et testé ultérieurement n'est plus capable de fixer le calcium correctement (exemples I et 3 du tableau I). Dans le cas contraire le RSF ne perd sa capacité à fixer le calcium qu'après 24 heures post mortem.

La suite de cette étude tentera de vérifier le bien-fondé de ces observations expérimentales.

\section{3. - Action du pH sur les propriétés du RSF de bovin}

Les incubations à différents $\mathrm{pH}$ sont faites à $30^{\circ} \mathrm{C}$. La figure 3 rapporte les résultats de l'expérience. Le captage de calcium est maximum à $\mathrm{pH} 7,2$. Il est totalement inefficace au-dessus de $\mathrm{pH} 7,7$ et au-dessous de 5,8. Ceci confirme bien les résultats 


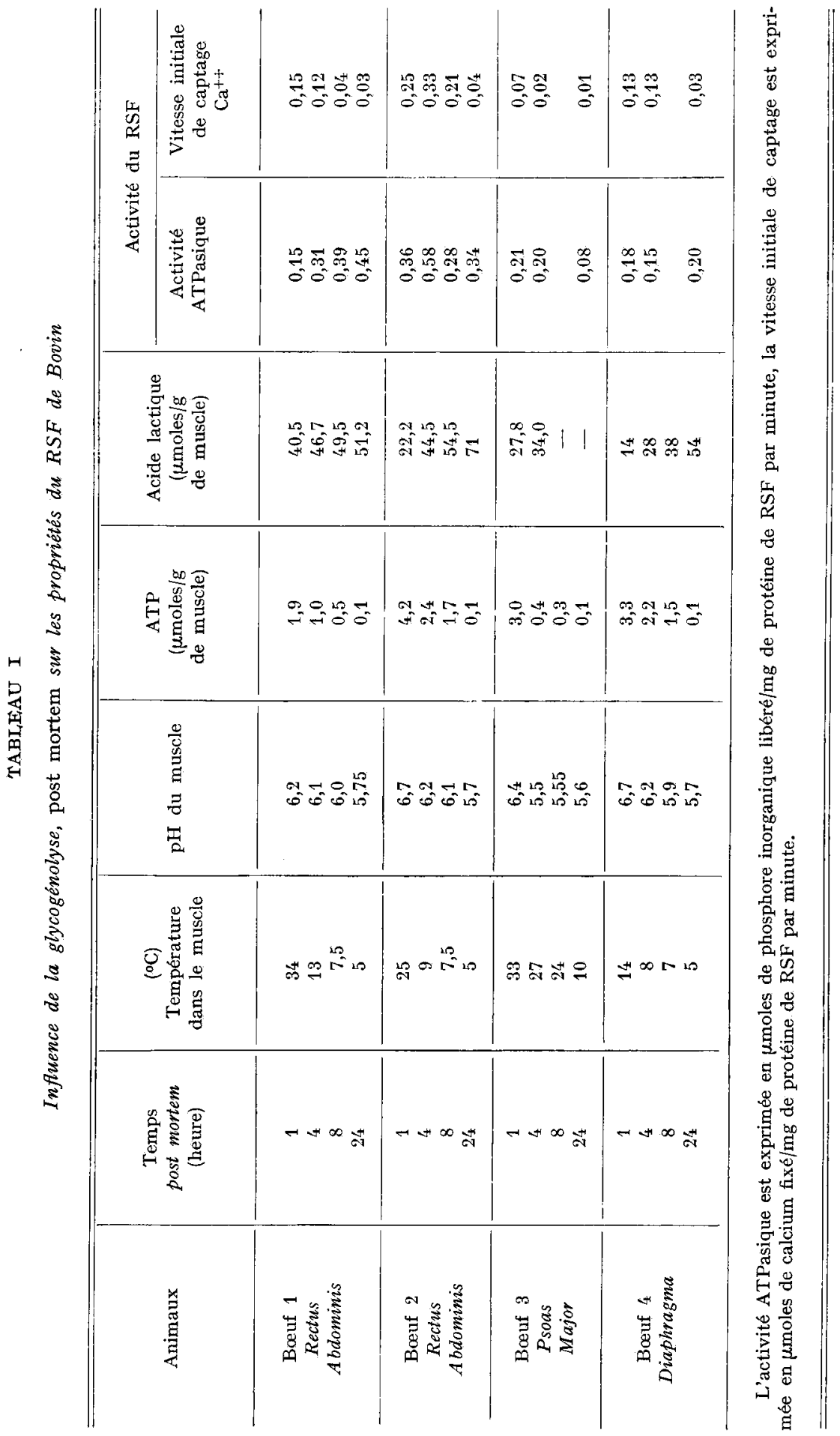




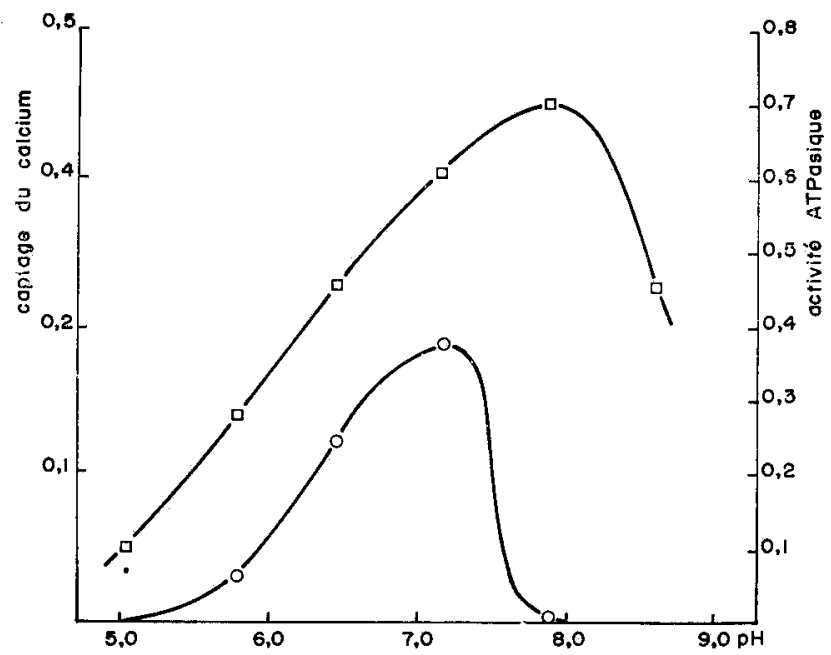

FIG. 3. - Action du pH sur le captage de calcium et sur l'activité ATPasique du RSF de Bovin

○- - - Le captage de calcium est exprimé en $\mu$ moles de calcium total capté par mg de protéines de granas et par minute

口- - L L L activité AT Pasique est exprimée en umoles de phosphore inorganique. libéré par $\mathrm{mg}$ de protéines de granas et par minute

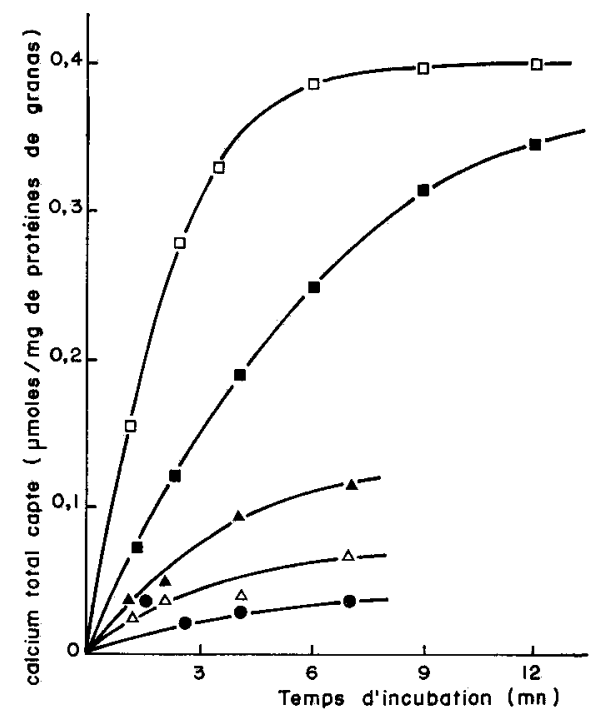

FIG. 4. - Captage de calcium à $p H 6,5$ et à différentes températures

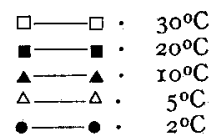


déjà obtenus sur RSF de porcs par GrEASER et al., en I967 et par CharpenTIER en 197 .

En outre, si l'activité ATPasique entre $\mathrm{pH}_{5,0}$ et 7,0 suit une évolution parallèle à celle de la vitesse de captage, elle est seulement diminuée de moitié à $\mathrm{pH} 6,0$ alors que la vitesse de captage n'est plus qu'à un quart de sa valeur maximale.

\section{4. - Action de la température à différents $p H$}

sur le captage de calcium par le RSF de muscle de bovin

La figure 4 montre la fixation de calcium par le $\mathrm{RSF}$ de bovin à $\mathrm{pH} 6,5$ et à $2^{\circ} \mathrm{C}, 5^{\circ} \mathrm{C}$, I0 $^{\circ} \mathrm{C}, 20^{\circ} \mathrm{C}$ et $30^{\circ} \mathrm{C}$. Il est possible de constater sur ces courbes l'intérêt de la vitesse initiale de captage pour apprécier l'état de fonctionnement des granas. En effet, le rapport $\frac{\text { activité à } 30^{\circ} \mathrm{C}}{\text { activité à } 20^{\circ} \mathrm{C}}$ est de I, I si on considère le maximum de calcium fixé alors qu'il est de 2,6 si on s'intéresse à la vitesse initiale de captage.

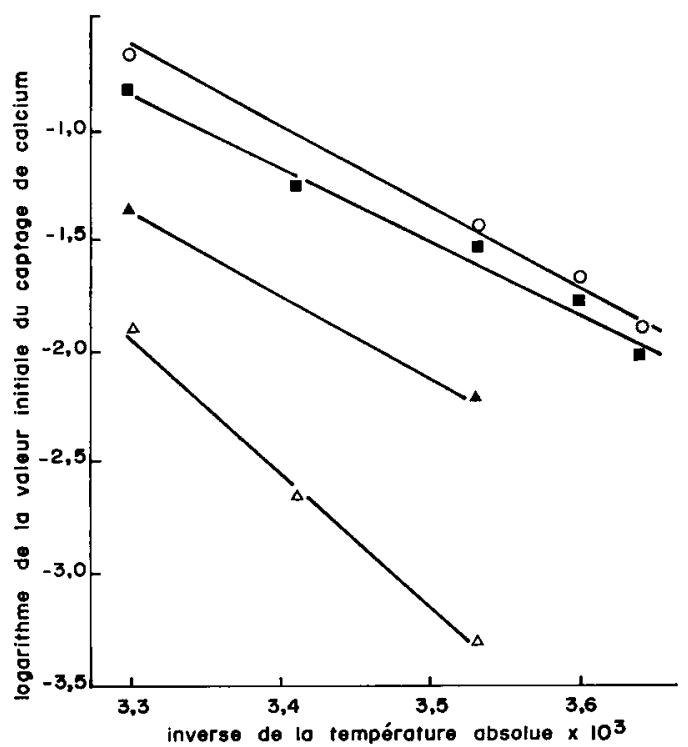

FIG. 5. - Action de la température sur le captage de calcium du RSF à différents pH

La valeur initiale du captage de calcium est exprimée en umoles de $\mathrm{Ca}^{++}$ par $\mathrm{mg}$ de protéines de granas et par minute

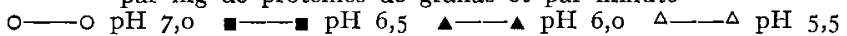

Les résultats obtenus sont résumés dans la figure 5 où le log de la vitesse initiale de fixation du calcium par le RSF est porté en fonction de l'inverse de la température absolue. Pour un $\mathrm{pH}$ donné, lorsque la température varie de $30^{\circ} \mathrm{C}$ à $5^{\circ} \mathrm{C}$ la vitesse de fixation du calcium est au moins divisée par le facteur Io. Ainsi à $\mathrm{pH} 7,0$ et à $2^{\circ} \mathrm{C}$ le RSF de bovin n'est pas plus efficace qu'il ne l'est à $30^{\circ} \mathrm{C}$ à $\mathrm{pH} 5,5$.

\section{5. - Influence de la conjonction $p H$-température sur le fonctionnement du RSF}

Dans les conditions habituelles de réfrigération des carcasses de bovin, et compte tenu de l'évolution du $\mathrm{pH}$ dans le muscle post mortem, il est possible de prévoir pour 
une température donnée les valeurs minimales et maximales possibles du $\mathrm{pH}$. Dans le but de reproduire in vitro les conditions limites de la pratique, le RSF du muscle Rectus abdominis de bovin est incubé pendant I heure à $30^{\circ} \mathrm{C}, 20^{\circ} \mathrm{C}$ ou $10^{\circ} \mathrm{C}$ dans du tampon Tris-maléate $\mathrm{o}, \mathrm{I} \mathrm{M}$ ajusté au $\mathrm{pH}$ correspondant aux valeurs extrêmes de cette fourchette pour chaque température choisie. Le RSF est ensuite repris par centrifugation à $30000 \mathrm{~g}$ pendant 30 minutes et remis en suspension dans du saccharose $0,25 \mathrm{M}$, histidine $5 \mathrm{mM}$. Ses propriétés de fixation du calcium et d'hydrolyse de 1'ATP sont alors testées dans les conditions optimales à $\mathrm{pH} 7,3$ et à la température de $30^{\circ} \mathrm{C}$.

\section{TABLEAU 2}

Infuence des conditions de $p H$ et de température existant dans une carcasse sur le fonctionnement ultérieur du RSF

\begin{tabular}{|c|c|c|c|c|c|c|}
\hline \multirow{3}{*}{$\begin{array}{l}\text { Températures } \\
\text { des incubations } \\
\text { préalables } \\
\left({ }^{\circ} \mathrm{C}\right)\end{array}$} & \multirow{2}{*}{\multicolumn{3}{|c|}{$\begin{array}{c}\text { Activité ATPasique } \\
\text { pH des incubations } \\
\text { préalables }\end{array}$}} & \multicolumn{3}{|c|}{ Captage de calcium } \\
\hline & & & & \multicolumn{3}{|c|}{$\begin{array}{l}\text { pH des incubations } \\
\text { préalables }\end{array}$} \\
\hline & 6,8 & 5,9 & 5,5 & 6,8 & 5,9 & 5,5 \\
\hline 30 & 452 & 452 & & 11,5 & $<1$ & \\
\hline 20 & 450 & & 128 & 2 & & $<1$ \\
\hline 10 & & 620 & $1 / 5$ & & $<1$ & $<1$ \\
\hline
\end{tabular}

L'incubation préalable en tampon Tris-maléate $0,1 \mathrm{M}$ est de 1 heure. L'activité ATPasique et le captage de calcium déterminés à $\mathrm{pH} 7,3$ et à $30^{\circ} \mathrm{C}$ sont exprimés en p. 100 par rapport au témoin n'ayant pas subi l'incubation préalable.

TABLEAU 3

Influence de la conjonction pH-température sur le fonctionnement ultérieur du RSF

\begin{tabular}{|c|c|c|c|c|c|c|c|c|}
\hline \multirow{3}{*}{$\begin{array}{c}\text { Incubations préalables } \\
\text { Températures } \\
\left({ }^{\circ} \mathrm{C}\right)\end{array}$} & \multicolumn{4}{|c|}{ Activité ATPasique } & \multicolumn{4}{|c|}{ Captage de Calcium } \\
\hline & \multicolumn{4}{|c|}{ pH des incubations préalables } & \multicolumn{4}{|c|}{ pH des incubations préalables } \\
\hline & 7,3 & 6,8 & 6,3 & 5,9 & 7,3 & 6,8 & 6,3 & 5,9 \\
\hline 40 & 311 & 778 & 834 & 722 & $<1$ & $<1$ & $<1$ & $<1$ \\
\hline 35 & 764 & 735 & 764 & & 50 & 32,7 & 20 & \\
\hline 30 & 321 & 485 & 357 & 536 & 55 & 35 & 35 & 15 \\
\hline
\end{tabular}

L'incubation préalable en tampon Tris maléate $0,1 \mathrm{M}$ est de 30 minutes. L'activité ATPasique et le captage de calcium déterminés à pH 7,3 et à $30^{\circ} \mathrm{C}$ sont exprimés en p. 100 par rapport au témoin n'ayant pas subi d'incubation préalable. 
Le tableau 2 rapporte les résultats obtenus dans cette étude. Il est net que très vite le RSF perd dans ces conditions sa capacité de fixer le calcium mais continue à hydrolyser 1'ATP de façon active sauf dans le cas des préincubations à $\mathrm{pH} 5,5$ où l'activité ATPasique reste sensiblement constante.

Ces résultats étant très différents de ceux obtenus par d'autres auteurs, une étude plus détaillée est faite selon le même protocole à 30,35 et $40^{\circ} \mathrm{C}$ et à des $\mathrm{pH}$ voisins de la neutralité, soit $7,3,6,8,6,3,5,9$. Le temps d'incubation est ramené à

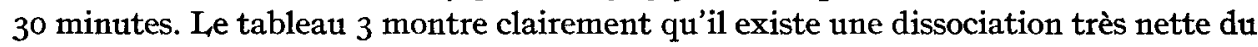
captage et de l'activité ATPasique. L'aptitude à fixer le calcium est complètement perdue après une incubation à $40^{\circ} \mathrm{C}$ quel que soit le $\mathrm{pH}$. Flle est mieux conservée à $30^{\circ} \mathrm{C}$ qu'à $35^{\circ} \mathrm{C}$. Elle décroît avec le $\mathrm{pH}$ des préincubations pour une température donnée. L'activité ATPasique, dans le même temps, se trouve multipliée par le facteur 3 à 8 . Ce facteur semble augmenter avec la diminution du $\mathrm{pH}$. En outre, par comparaison des tableaux 2 et 3 , il est possible d'apprécier l'influence du temps de préincubation à un $\mathrm{pH}$ et à une température donnés. Ainsi à $\mathrm{pH} 6,8$ et à $30^{\circ} \mathrm{C}$, une préincubation de 30 minutes permet de conserver 35 p. roo du captage initial. Dans le tableau 2, le temps de préincubation est doublé, le captage n'est plus que de II, 5 p. 10o. Cette dégrađation irréversible du RSF évolue en fonction du temps dans le même sens à $\mathrm{pH}$ 5,9.

TABLEAU 4

Influence de la conjonction pH-température à pression osmotique isotonique sur le fonctionnement ultérieur du RSF

\begin{tabular}{|c|c|c|c|c|c|c|}
\hline \multirow{2}{*}{$\begin{array}{c}\text { Incubations } \\
\text { préalables }\end{array} \begin{cases}\mathrm{pH} & \ldots \\
{ }^{\circ} \mathrm{C} & \ldots \ldots\end{cases}$} & \multicolumn{2}{|c|}{7,3} & \multicolumn{2}{|c|}{6,3} & \multicolumn{2}{|c|}{5,6} \\
\hline & 40 & 30 & 20 & 10 & 20 & 10 \\
\hline Activité ATPasique.... & 330 & 230 & 150 & 120 & 130 & 120 \\
\hline Captage de calcium.... & $<1$ & 50 & 4 & $<1$ & $<1$ & $<1$ \\
\hline
\end{tabular}

L'incubation préalable est faite en tampon Tris-maléate 0,1 M, saccharose $0,25 \mathrm{M}$ pendant 2 heures. L'activité ATPasique et le captage de calcium déterminés à $\mathrm{pH} 7,3$ et à $30^{\circ} \mathrm{C}$ sont exprimés en p. 100 par rapport au témoin n'ayant pas subi d'incubation préalable.

Il a paru intéressant de répéter ces expériences en se plaçant à pression osmotique isotonique. Des essais d'incubation préalable dans du tampon Tris-maléate o, I M auquel il est ajouté du saccharose $0,25 \mathrm{M}$ montrent que le RSF de muscle Rectus abdominis de bovin se comporte alors d'une façon beaucoup plus résistante. Ainsi une préincubation de 30 minutes à $\mathrm{pH} 7,3$ et à $30^{\circ} \mathrm{C}$ ne provoque aucune dénaturation du RSF. Cependant, en augmentant le temps de séjour à 2 heures dans ces conditions, (tableau 4), il est possible de retrouver le même phénomène. Seule l'hydrolyse de l'ATP semble alors rester dans des limites comparables à celles du témoin. 


\section{6. - Action du pH sur l'activité protéolytique des suspensions de RSF}

Un test simple d'activité protéolytique en milieu acétique à pH 3,5 montre que les extraits de granas contiennent une fraction importante des lysosomes du muscle. Ceci a conduit à établir un spectre d'activité protéolytique aux $\mathrm{pH}$ employés dans les incubations préalables. Une fraction de $\mathrm{I} \mathrm{ml}$ de suspension de RSF est ajoutée à I $\mathrm{ml}$ de tampon phosphate $0,2 \mathrm{M}$ ajusté au $\mathrm{pH}$ désiré. Les protéines de la suspension servent de substrat, soit environ $5 \mathrm{mg} / \mathrm{ml}$. Pour chaque $\mathrm{pH}$ un témoin est stoppé au temps o par addition de $\mathrm{I} \mathrm{ml}$ d'acide trichloracétique $\mathrm{I} 5 \mathrm{p}$. Ioo et gardé à la température ambiante. Les essais sont incubés à $35^{\circ} \mathrm{C}$ pendant 6 heures, stoppés par addition

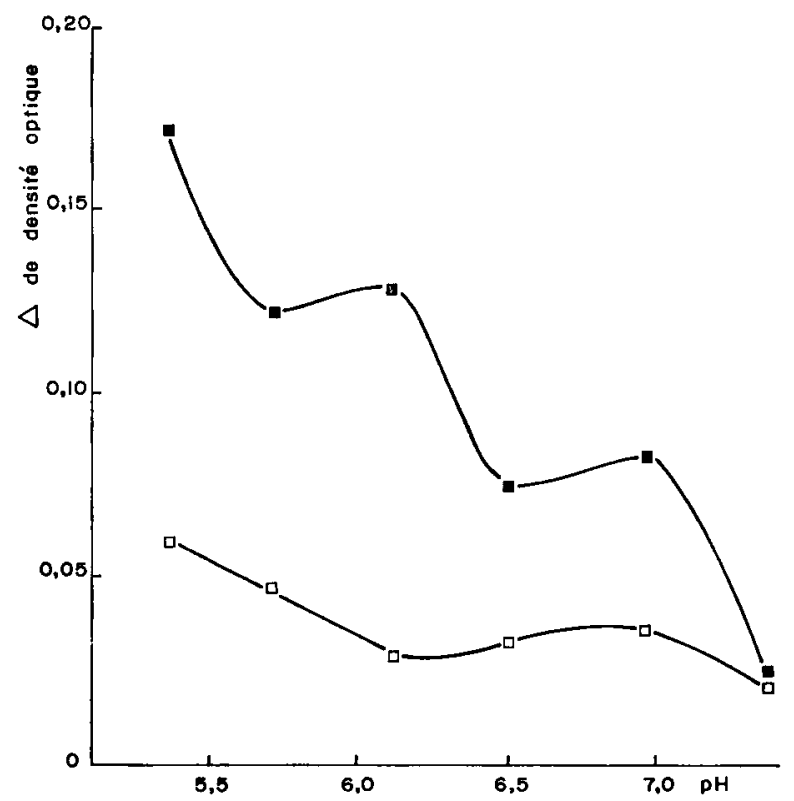

FIG. 6. - Spectre d'activité protéolytique des extraits de RSF de Bovin en fonction du $p H$

- - Coloration à la ninhydrine

口- $\square \cdot$ Réaction de Folin

d'acide trichloracétique, et centrifugés en même temps que les témoins. Des frottis sur lame, colorés au bleu de méthylène n'ont révélé aucune culture microbienne visible en microscopie optique. Une coloration de Folin et une réaction à la ninhydrine sont faites sur chaque surnageant. La différence de densité optique entre le témoin et l'essai est portée en fonction $\mathrm{du} \mathrm{pH}$ dans la figure 6 . Il est ainsi possible de voir qu'une hydrolyse des protéines existe et va en décroissant de $\mathrm{pH} 5,5 \mathrm{à} \mathrm{pH} 7,0$. Il est donc probable que la protéolyse s'ajoute à l'action de la température et du $\mathrm{pH}$ pour dénaturer les membranes de RSF de muscles de bovins. 


\section{DISCUSSION}

Le RSF de bovins se comporte de façon analogue à celui de lapin ou de porc dans des conditions similaires d'incubation. Les concentrations de $\mathrm{Mg}^{++}$, de calcium liōre, d'A'TP et d'oxalate semblent agir dans le même sens sur l'activité ATPasique et le captage net, donc sur la somme algébrique des flux sortant et entrant de calcium à travers les membranes. Cependant la vitesse initiale de captage par mg de protéines de granas prélevés le plus tôt possible après la mort est quelquefois très différente de celles mentionnées par d'autres auteurs. Ainsi TAKaUjI, Yamamoto et NaGAI (I967) obtiennent une vitesse initiale de $0,05 \mu \mathrm{mole} / \mathrm{mg}$ de protéine/minute bien que leurs conditions d'incubations soient analogues aux nôtres. La vitesse initiale de captage obtenue par INESI et WATANABE (I967) atteint I $\mu$ mole de $\mathrm{Ca}^{++} / \mathrm{mg}$ protéine/minute en incubant du RSF de muscle de lapin en présence de calcium Ioo $\mu \mathrm{M}$ à $20^{\circ} \mathrm{C}$. Bien que la provenance du RSF d'espèces, d'individus ou de muscles divers puisse influer, il semble que les différences dans les activités spécifiques observées soient dues à la proportion de granas en bon état de fonctionnement tel que l'ont mis en évidence GREASER et al. (Ig69 a).

L'étude de l'action de la température sur la fixation du calcium par le RSF de bovin extrait aussitôt après l'abattage montre bien qu'au-dessous de ro $0^{\circ} \mathrm{C}$, même à $\mathrm{pH} 7,0$, le réticulum est incapable de refixer efficacement le calcium libre, même en présence d'une concentration importante d'ATP. Ceci peut contribuer à vérifier l'hypothèse selon laquelle la contracture provoquée par le froid est due à l'inhibition du système relaxant alors que les myofibrilles ont encore la possibilité de fonctionner. On constate également que lorsque le $\mathrm{pH}$ atteint approximativement la valeur de 5,9 le réticulum sarcoplasmique n'est plus capable de capter normalement le calcium libre. Celui-ci peut alors diffuser dans la cellule et provoquer une réaction des protéines contractibles si la concentration en ATP et la température du sarcoplasme le permettent. Les résultats confirment ceux de CHARPEN'TIER (I97I) obtenus sur RSF de porc. En outre, le séjour dans un environnement agressif provoque une dénaturation irréversible du RSF.

Le calcul de la force ionique tenant compte de la molarité et des $\mathrm{pK}$ des tampons employés dans les incubations préalables donne les valeurs $0, \mathrm{II}, 0, \mathrm{I} 2,0, \mathrm{I} 3$ et $0, \mathrm{I} 4$ pour les $\mathrm{pH}$ respectifs de $5,5,6,0,6,5,7,0$. Il est donc possible de comparer les résultats obtenus aux tableaux 2 et 3 avec ceux de CHARPENTIER (I97I) et GREASER et al. (I969 $b$ ) qui ont travaillé sur le RSF de porc. CHARPENTIER trouve après I heure de préincubation à $40^{\circ} \mathrm{C}$ et à $\mathrm{pH} 7,3$ un captage de caicium pratiquement équivalent au témoin. GREASER et al., obtiennent 95 p. IOO, 55 p. IOO, 5 p. IOO de la capacité initiale à accumuler le calcium après incubation préalable d'une heure à $\mathrm{pH} 5,6$ et aux températures de $0^{\circ} \mathrm{C}, 25^{\circ} \mathrm{C}$ et $37^{\circ} \mathrm{C}$. Leurs préincubations d'une heure à un $\mathrm{pH}$ supérieur à 6,0 , quelle que soit la température, ne dénaturent pratiquement pas les microsomes. Les résultats tout à fait différents présentés ici permettent d'avancer deux hypothèses : soit le réticulum sarcoplasmique de muscle de bovin est plus fragile que celui du porc, soit un facteur autre que le $\mathrm{pH}$, la température ou la force ionique accélère la dégradation du RSF de bovin. 
Or la figure 6 montre dans les suspensions de granas de bœuf l'existence d'une activité protéolytique provenant très certainement des lysosomes entraînés en même temps que le RSF lors des centrifugations fractionnées. Cette protéolyse augmente avec l'acidité du milieu. Il est intéressant de remarquer dans les tableaux 2 et 3 les valeurs exceptionnelles de l'activité ATPasique liée à des vitesses de captage très faibles. Il semble que l'hydrolyse de l'ATP par le RSF se maintient à un niveau très élevé analogue à celui qu'il est possible d'observer pendant les deux premières minutes de captage (figure 2). Les incubations réalisées en tampon isotonique, permettant de se rapprocher des conditions intracellulaires, (tabl. 4) dénaturent moins les granas. Le même phénomène reste cependant visible. La dissociation dans le RSF du transport de calcium et de 1'hydrolyse de l'ATP activée par le calcium a été observée de nombreuses fois. MARTONOSI (I969) a mis en évidence que le transport du calcium à travers la membrane du réticulum sarcoplasmique se fait par l'intermédiaire d'une protéine phosphorée et qu'il est possible par inhibition du captage d'accumuler cette protéine dans les membranes. Sarzala et Drabikowski (I969) ne trouvent pas d'activité protéolytique dans les granas gardés à $0^{\circ} \mathrm{C}$ mais ils mettent en évidence une libération d'acides gras. Ces acides gras libres inhibent le captage du calcium et augmentent l'hydrolyse de l'ATP. Enfin MaR'Tonosi ( $1968 b$ ) montre que les microsomes traités par la phospholipase $C$ perdent leur capacité de fixer le calcium et d'hydrolyser l'ATP. Par contre, lorsqu'il fait agir la trypsine il observe des résultats tout à fait comparables à ceux obtenus dans cette étude.

\section{CONCLUSION}

Il semble bien démontré que la capacité à accumuler le calcium du réticulum sarcoplasmique du muscle de bovin diminue en fonction du temps post mortem. Il se confirme de plus que cette dénaturation est essentiellement provoquée par la conjonction $\mathrm{pH}$ acide-température élevée dans le muscle mais il n'est peut-être pas exclu que les enzymes hydrolytiques acides du muscle contribuent à l'altération post mortemdu fonctionnement du RSF dès les premiers instants qui suivent l'abattage.

La contracture provoquée par le froid peut être en partie expliquée par l'inhibition du réticulum sarcoplasmique aux basses températures ce qui entraîne un relargage du calcium dans un système pourvu en ATP donc contractile. Pour éviter qu'une contraction n'ait lieu avant l'installation de la rigor mortis il est nécessaire d'éviter le relargage trop préocce du calcium ce qui dans l'état actuel des connaissances et sur un plan pratique ne peut s'effectuer qu'en évitant de placer les carcasses de bovin dans des conditions de froid trop rigoureuses pendant les instants qui suivent 1'abattage.

Reçu pour publication en avril 1971. 


\section{SUMMARY}

\section{EFFECT OF PH AND TEMPERATURE POST MORTEM ON UPTAKE OF CALCIUM AND ADENOSINE TRIPHOSPHATASE ACTIVITY} OF THE SEPARATED SARCOPLASMIC RETICULUM OF MUSCLE OF CATTLE

The object of this study was to define the effects of $\mathrm{pH}$ and temperature on activity of the separated sarcoplasmic reticulum of the rectus abdominis muscle of cattle.

- In the presence of magnesium, ATP and oxalate the separated sarcoplasmic reticulum or grana could fix all the calcium of the solution in 2 to 3 minutes. The ATP-ase activity diving uptake was 5 times greater than in absence of uptake (fig. I and 2 ).

- The uptake of calcium by the grana decreased regularly with acidification of the muscle post mortem, while ATPase activity remained at a constant level (table $\mathrm{I}$ ).

- Fixation of calcium was completely inhibited by pH below 5.8 or above 7.7 (fig. 3).

- From study of the effect of temperature it could be shown that below $5^{\circ} \mathrm{C}$ there was no longer uptake of calcium at any $\mathrm{pH}$ (fig. 4 and 5).

- Incubation of the separated sarcoplasmic reticulum in o.I $\mathrm{M}$ trimaleate buffer at temperatures between $10^{\circ}$ and $40^{\circ} \mathrm{C}$ and at $\mathrm{pH}$ between 5.5. and 7.3 inhibited the uptake of calcium and increased the hydrolysis of ATP $3^{-}$to 8-fold (tables 2 and 3 ). The ability to fix calcium was

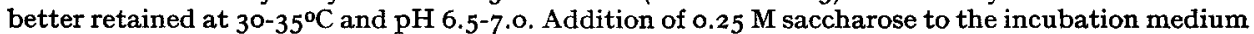
allowed the retention of ATP-ase activity of the separated sarcoplasmic reticulum and reduced the inhibition of capacity to accumulate calcium. The effect of proteolytic activity during the incubation is discussed.

\section{RÉFÉRENCES BIBLIOGRAPHIQUES}

Bergmeyer H. U., 1963. Methods of Enzyme Analysis. Academic Press, New York.

Charpentier J., I97I. Caractéristiques biochimiques du réticulum sarcoplasmique du muscle de Porc post mortem. Ann. Biol. anim. Biochim. Biophys., (sous presse).

EвASHi S., I961. Calcium Binding Activity of Vesicular Relaxing Factor. J. Biochem., 50, $236-244$.

Greaser M. L., Cassens R. G., Hoekstra W. G., I967. Changes in Oxalate-Stimulated Calcium Accumulation in Particulate Fraction from Post mortem Muscle. J. A gr. Food Chem., 15, III2-III7.

Greaser M. L., Cassens R. G., Hoemstra W. G., Briskey E. J., I969a. Purification and Ultrastructure Properties of the Calcium Accumulating Membranes in Isolated Sarcoplasmic Preparations from Skeletal Muscle. J. Cell. Physiol., 74, 37-50.

Greaser M. L., Cassens R. G., Hoekstra W. G., Briskey E. J., I969 b. The effect of pH Temperature Treatments on the Calcium Accumulating Ability of Purified Sarcoplasmic Reticulum. J. Food Sci., 34, 633-637.

Harigaya S., Ogawa Y., Sugita H., I968. Calcium Binding Activity of Microsomal Fraction of Rabbit Red Muscle. J. Biochem., 63, 324-33I.

Hasselbach W., Makinose M., I96r. Die Calcium pumpe der Erschlaffungsgrana des Muskels und Ihre Abbängigkeit von der ATP-Spalting. Biochem. Z., 333, 518-528.

Inesi G., Watanabe S., 1967. Temperature Dependance of ATP Hydrolysis and Calcium Uptake by Fragmented Sarcoplasmic Membranes. Arch. Biochem. Biophys., 121, 665-67I.

Martonosi A., Feretos R., 1964. Reticulum sarcoplasmic. I. The uptake of $\mathrm{Ca}^{++}$by Sarcoplasmic Reticulum Fragments. J. Biol. Chem., 239, 648-658.

Martonosi A., I968. Sarcoplasmic Reticulum. IV. Solubilization of Microsomal Adenosine Triphosphatase. J. Biol. Chem., 243, 6r-70.

Martonosi A., I969. Sarcoplasmic Reticulum. VII. Properties of phosphoprotein Intermediate Implicated in Calcium Transport. J. Biol. Chem., 244, 613-62o.

Samaha F. J., Gergely J., I965. Ca++ Uptake and ATPase of Human Sarcoplasmic Reticulum. $J$. Clin. Invest., 44, 1425-I43I.

Sarzala M. G., Drabikowski W., I969. Free Fatty Acids as a Factor Modifying Properties of Fragmented Sarcoplasmic Reticulum During Ageing. Life Sci., 8, part II, 477-483.

Szent-György, 1947. Chemistry of Muscular Contraction. Academic Press. N. Y., Ireéd.

Takauji M., Yamamoto T., Nagai T., r967. On the Relaxing Factor System in Rabbit Red Muscle. Jap. J. Physiol., 17, III-121. 